\title{
Influence of turbulence-radiation interaction on radiative heat transfer to furnace wall and temperature distribution in large-scale industrial furnaces enveloping hydrocarbon flame
}

\author{
Seiichi TAKEUCHI*, Shinichi ASAO* and Masashi YAMAKAWA** \\ * Department of Mechanical Engineering, College of Industrial Technology \\ 1-27-1 Nishikoya, Amagasaki-shi, Hyogo 661-0047, Japan \\ E-mail: takeuchi@cit.sangitan.ac.jp \\ ** Department of Mechanical and System Engineering, Kyoto Institute of Technology \\ Goshokaido-cho, Mastugasaki, Sakyo-ku, Kyoto 606-8585, Japan
}

Received: 6 May 2021; Revised: 14 June 2021; Accepted: 29 June 2021

\begin{abstract}
Theoretical examinations based on absorption line databases were carried out to investigate the influence of turbulence-radiation interaction on the radiative heat transfer arriving at the wall of large-scale industrial furnaces including hydrocarbon flame, where the re-absorption of radiative energy by combustion gas on its path toward objects to be heated cannot be neglected. In this study, we combined an improved version of our previous method for reducing the calculation load required for tracing turbulent fluctuation in temperature in great detail and an efficient method proposed in our previous papers to reduce the enormous calculation load contingent on detailed non-gray analysis. When we combined these methods with a governing equation solver for obtaining the spatial distribution of time-averaged values of temperature, concentration, velocity, and so on, we could evaluate the heat transfer including radiation in large-scale industrial furnaces enveloping turbulent hydrocarbon flame with sufficient accuracy equivalent to Line-by-Line analysis and with a feasible calculation load. Our application of this calculation method to large-scale furnaces enveloping hydrocarbon flame revealed that neglecting the turbulence-radiation interaction in numerical simulation gave rise to an obvious change in the heat flux distribution on the side wall and in the spatial distribution of the time-averaged temperature. In addition, change in the total amount of radiative energy arriving at the side wall caused by neglecting the turbulence-radiation interaction was fairly small compared with the change observed in our previous report on a model optical path imaging the typical course of radiative energy in large-scale industrial furnaces fueled by propane.
\end{abstract}

Keywords: Thermal radiation, Turbulent combustion, Numerical simulation, Radiative wall heat flux, Large-scale industrial furnaces, Turbulence-radiation interaction, Hydrocarbon flame

\section{Introduction}

The performance of heat transfer to a heated product is the main performance index of a heating furnace or industrial combustion furnace (i.e., a boiler). The large-scale industrial furnaces used at steel plants and thermal power plants tend to be served as build-to-order production. Since it is quite expensive to construct a furnace on the system scale for experiments on heat transfer performance, being able to predict the heat transfer situation accurately by a numerical simulation is strongly desired.

In the heat transfer of a combustion furnace, the temperature gradient becomes gentle so that the scale of the flame becomes large, and the heat flux caused by the temperature gradient decreases. On the other hand, the radiative heat flux to the outside of a gas layer increases so that the gas layer becomes thick. As such, in a large-scale combustion 
furnace, the radiative heat transfer inevitably becomes dominant and occupies most of the heat transfer amount. Therefore, it is important that the radiative heat transfer be properly predicted by a numerical simulation.

The flame formed in a large-scale industrial furnace is a turbulent flame in which the temperature and gas compositions are sharply changed in almost all cases. Therefore, numerical simulation of radiative heat transfer in a large-scale furnace should be performed on the basis of the spatial distribution of the temperature and gas compositions of each moment. However, since the simulation is a practical numerical analysis for supporting the furnace design, ideally we want to reduce the calculation load. To enable this, two calculation methods are usually combined: one, a method of acquiring the time-averaged spatial distributions of the temperature and gas composition of a turbulent flame by using Reynolds-averaged Navier-Stokes equations (RANS), and two, a method of calculating the radiative heat transfer based on the time-averaged spatial distributions of temperature and gas composition.

However, the emission power of a black body shows strong nonlinearity to temperature. Therefore, with something like a turbulent flame, where the temperature fluctuation is large, there is a high possibility that the emission energy calculated from the time-averaged temperature will disagree with the value of the time-averaged emission energy. Moreover, a great part of the emission energy is absorbed once on the optical path toward heated products arranged near the furnace wall. Also, since the spectrum of the absorption coefficient of gas on the optical path depends on both the temperature and the gas composition related to the temperature, there is a possibility that the amount of absorption depends on the correlation of the temperature between the emitted gas and the absorbed gas. For these reasons, radiative heat transfer predicted by using practical numerical analysis based on the time-averaged spatial distributions of temperature and gas composition is far from accurate. This problem of how turbulent fluctuation influences thermal radiation is called turbulence-radiation interaction (TRI). TRI and the generally low accuracy of gas radiation models expressing the non-gray characteristics of infrared activated gas are recognized as the two major factors that lower the reliability of the numerical simulations of radiative heat transfer in a furnace.

Modest et al. have summarized the details of TRI in their book (Modest and Haworth, 2016). As for the numerical simulation of the turbulent combustion field taking TRI into consideration, studies on performing Large Eddy Simulation (LES) in consideration of TRI began to appear from around 2009 (Chandy et al., 2009; Gupta et al., 2009, 2013; Coelho, 2009). Some studies (Poitou et al., 2011, 2012) have analyzed the turbulent combustion field by using a method that combines LES and the Discrete Ordinate method (DOM), and another study (Somnath et al., 2014) obtained the radiative heat transfer from the supersonic water vapor flow in a pipe that is not a combustion field. However, other studies (Roger et al., 2009, 2011) have pointed out a defect of the Sub-Grid Scale model used for TRI, and it is difficult to clarify exactly how LES in consideration of TRI are constructed. There have been recent efforts to come up with a new calculation method for determining the radiative heat transfer in consideration of TRI combined with LES by using the Monte Carlo method (Rejeb and Echekki, 2017). However, this line of inquiry does not consider a large area, such as an industrial combustion furnace. Also, it seems that the calculation load is too high to use LES in consideration of TRI as a design support tool for an industrial furnace even if the Sub-Grid Scale model or various new calculation methods are constructed.

To enable practical calculation, we need a method that can reduce the calculation load more significantly than the studies discussed above. There is a method of solving the time-averaged governing equation, which introduces a turbulent model (such as RANS) and deals with turbulent fluctuation by using a probability density function (PDF) (Mazumder and Modest, 1999). There are also several studies that have examined the influence of TRI by using this method (Coelho et al., 2003; Habibi et al., 2007), and the effectiveness of the method has been demonstrated. However, it seems that its calculation load is quite high, as it is necessary to carry out the calculation of radiative heat transfer in consideration of non-gray characteristics for every combination of the various kinds of fluctuation value expressed in the PDF. If we can, slightly alter the calculation of radiative heat transfer based on a time-averaged value, it should be possible to come up with a calculation method that delivers results equal to those obtained by calculating the radiative heat transfer with tracing turbulent fluctuation.

As indicated above, there has been a considerable amount of research on TRI. However, aside from our own prior work on turbulent hydrogen flame (Takeuchi and Okamoto, 2017), there is almost no knowledge about the influence of TRI on the radiative heat transfer to the side wall of a large-scale industrial furnace, as most previous studies have focused on a small flame of the laboratory scale. Moreover, the focus of the previous research has been limited to the temperature reduction of the flame by thermal radiation, or to the composition of the chemical species influenced by temperature change, or to the generation of soot, and so on. For example, the fuel nozzle diameter of the Sandia Flame 
D (TNF workshop, 2021), which most studies related to TRI have used, is just $9 \mathrm{~mm}$. The radiative heat transfer is remarkably small compared with the convective heat transfer in such a small flame. Moreover, because the percentage that is re-absorbed by infrared activated gas, which exists inside or outside of the flame over the emission energy, is only $1 \%$, it would make sense in such cases if TRI in the absorption process was not considered at all. On the other hand, because the percentage that is absorbed before the emission energy reaches a water tube arranged near the furnace side wall extends dozens of percent, it is necessary in these cases to consider TRI in the absorption process. In addition, when TRI in the absorption process is dealt with, it is necessary to consider the correlation of fluctuations of both absorption coefficients of the emitted gas and the absorbed gas linked with the temperature fluctuation. As such, TRI in the absorption process is quite a lot more complicated than it is in the emission process. In a large-scale furnace where consideration of TRI in the absorption process is necessary, it would have technological benefits if we could clarify the influence TRI exerts on the amount of radiative heat transfer to the heated product from a turbulent flame. It would also be beneficial to clarify the extent to which the obtained results need to be corrected by calculating the radiative heat transfer based on a time-averaged value. However, virtually no studies have considered the influence of TRI from this point of view.

Moreover, in the study of TRI, the temperature dependencies of a Planck function and an absorption coefficient spectrum are taken into consideration in addition to the temperature fluctuation history, as pointed out in a prior report (Li and Modest, 2003). However, as mentioned above, the precision of gas radiation models expressing the non-gray characteristics of infrared activated gas are generally low. For example, the Exponential Wide-Band model is $40 \%$ less accurate than the actual measurement of emissivity based on the condition of temperature and gas thickness (Edwards, 1976). When gas radiation models are adopted in the examination of TRI, the influence of TRI and the error caused by the gas radiation models will mix, and this obviously needs to be avoided.

In addition, as mentioned above, when dealing with TRI in the absorption process, it is necessary to consider the correlation of fluctuations of both the absorption coefficients of the emitted gas and the absorbed gas linked with the temperature fluctuation. To obtain a stable result statistically, we need to trace the turbulent fluctuation history over an enormous number of time steps, and a calculation in addition to the one performed by the gas radiation model is needed for each one. When Line-by-Line analysis (LBL) is adopted, which is a detailed-non-gray analysis with a very high calculation load, the calculation of radiative heat transfer in consideration of TRI focused on an entire large-scale furnace becomes impossible to carry out not only in terms of practical numerical analysis but also in terms of numerical analysis of the study purpose.

To resolve the above issues, we previously proposed a method for treating the complicated spectrum of absorption coefficients and another method for handling turbulent fluctuations in temperature and in partial pressure of infrared-active gas in order to enable a fairly accurate simulation with a feasible calculation load in relation to the radiative heat transfer in a large-scale industrial furnace. We also examined how TRI affects the amount of radiative heat transfer arriving at the side wall of a large-scale furnace. In our previous study, we focused on hydrogen flames. However, since hydrocarbon is usually used as a fuel in industrial furnaces, not hydrogen, there is a problem in that it lacks relevance to public usage. We therefore adapted our method (Takeuchi and Okamoto, 2020) so that it could be applied to the radiative heat transfer in the large-scale hydrocarbon flame formed in industrial furnaces. Validity tests of the revised method showed that its error rate was satisfactorily small. In the present study, we applied our improved calculation method to the radiative heat transfer in a large-scale furnace containing hydrocarbon flame to examine the influence of TRI on the radiative heat transfer arriving at the side wall of a large-scale furnace. We also investigated the mechanism underlying the influences.

\section{Application of improved calculation method for turbulent hydrocarbon flame to the radiative heat transfer in whole furnace}

As described in the introduction, when the analysis of radiative heat transfer that considers TRI focused on an entire large-scale furnace is performed, there are problems in terms of both the enormous amount of bandwidth divisions contingent on detailed non-gray analysis (e.g., LBL) and the enormous calculation load required for tracing turbulent fluctuations. Therefore, there is a need for a calculation method that can deliver fairly accurate results obtained with a feasible calculation load. Previous papers have proposed a method that reduces the enormous calculation load contingent on detailed non-gray analysis (Takeuchi and Okamoto, 2012a, 2012b), and its validity has 
been confirmed for both hydrogen flames and hydrocarbon flames. However, the method we previously proposed for reducing the calculation load required for tracing turbulent fluctuations in temperature in great detail cannot give satisfactory results in relation to the large-scale hydrocarbon flame (Takeuchi and Okamoto, 2013). Therefore, in a recent paper (Takeuchi and Okamoto, 2020), we proposed an improved method for reducing the calculation load associated with a detailed trace of turbulent fluctuation and examined its validity when applying it to the large-scale hydrocarbon flame. Results showed that the error rate of the improved method is sufficiently smaller than that when the turbulent fluctuation of temperature and gas composition is entirely disregarded. Moreover, this improvement related to the treatment of turbulent fluctuation is satisfactorily valid even if coupled with our method for treating the complicated spectrum of absorption coefficients. Below, we describe the details of combining the above two methods to the simulation of radiative heat transfer in large-scale furnaces enveloping turbulent hydrocarbon flames.

First, in this study, we targeted the turbulent hydrocarbon diffusion flame formed by the combustion of propane under atmospheric pressure, as in our previous study (Takeuchi and Okamoto, 2020). In terms of infrared-active gas, because the importance of $\mathrm{CO}$ is relatively low as an emission source and as an absorption body, we removed $\mathrm{CO}$ as a target of consideration and treated only $\mathrm{H}_{2} \mathrm{O}$ and $\mathrm{CO}_{2}$. The absorption coefficients of $\mathrm{H}_{2} \mathrm{O}$ and $\mathrm{CO}_{2}$ were calculated from an absorption line database that combined HITRAN 2008 / 2009 update (Rothman, 2020) and HITEMP 2010 (Rothman, 2010). Here, the partial pressures of $\mathrm{H}_{2} \mathrm{O}$ and $\mathrm{CO}_{2}$ influence the bandwidth of an absorption line and the number density of each molecule, respectively. In the turbulent diffusion flame, the partial pressures of gases are related to the temperature, and the method of relating the partial pressures of gases to the temperature follows a method of a previous report (Takeuchi and Okamoto, 2020). The basic principle of the above method is as follows. First, the turbulent transport coefficients of chemical species and an enthalpy are assumed to be the same on the basis of an empirical rule. When influences of radiative heat transfer are disregarded, a mixture fraction is acquired from a specific enthalpy. When the temperature and the partial pressures of gases that satisfy a specific enthalpy and a mixture fraction are acquired, the combustion reactions are assumed to be mixed-control. In other words, it is assumed that $\mathrm{O}_{2}$ does not exist inside the flame and propane does not exist outside the flame. The temperature and the partial pressures of gases inside and outside of each flame are acquired while changing a specific enthalpy little by little. This relation is finally rearranged as a relation between the temperature and the partial pressures of gases, as shown in Fig. 1.

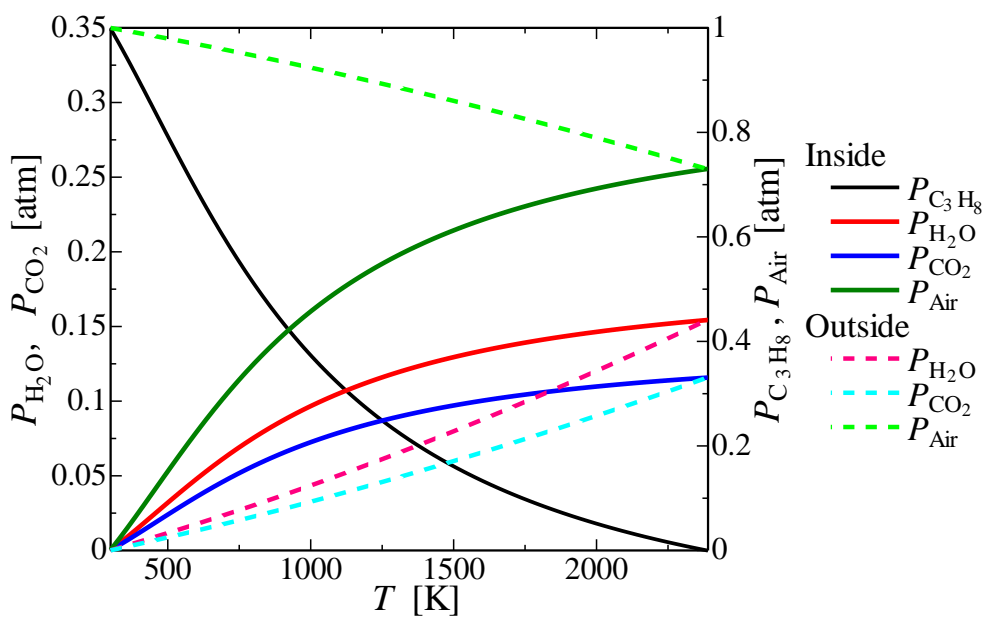

Fig.1 Relationships among temperature and partial pressures of several gaseous components.

We discuss later how to discriminate the inside and outside of the flame when calculating the radiative heat transfer focused on an entire large-scale industrial furnace. From the relationship indicated in Fig. 1, once the temperature has been decided, the various partial pressures of gases are decided, along with the bandwidths of the absorption lines. Therefore, the spectra of absorption coefficients are decided as a function only of the temperature. For this calculation, the spectra of the absorption coefficients of $\mathrm{H}_{2} \mathrm{O}$ and $\mathrm{CO}_{2}$ are respectively acquired by the same method and then added to each other. Moreover, the bandwidth of the wave number of $0-10000 \mathrm{~cm}^{-1}$ is divided every $0.01 \mathrm{~cm}^{-1}$, and $1,000,000$ discrete spectra of absorption coefficients are acquired.

We applied our method for treating complicated spectra of absorption coefficients as follows. First, the whole band 
of $0-10000 \mathrm{~cm}^{-1}$ was shared with 100 narrow bands $100 \mathrm{~cm}^{-1}$ width. Next, the spectra of the absorption coefficients in the narrow band of $100 \mathrm{~cm}^{-1}$ width were sorted in descending order. After that, they were approximated by the weighted sum of 24 elementary gray gases, and the bandwidth of the $i$-th elementary gray gas in the narrow band was acquired by the easy secondary function, as

$$
(\Delta \eta)_{i}=0.02 i^{2} \quad\left[\mathrm{~cm}^{-1}\right] \quad \text { (fraction } 2 \mathrm{~cm}^{-1} \text { is added to the } 24 \text { th elementary gray gas) }
$$

This method of bandwidth division in the narrow band was applied while the whole temperature range was unified. In the calculation of radiative heat transfer in non-isothermal fields, the absorption coefficient of the $i$-th elementary gray gas on the absorption side are made to correspond to the emission energy from the $i$-th elementary gray gas on the emission side. After reducing the calculation load contingent on bandwidth division in this way, we combined this method with our improved method for reducing the calculation load associated with the detailed trace of turbulent fluctuation. Working on the assumption that temperature fluctuations of two arbitrary positions are independent from each other, we treated the temperature fluctuation of the emitted gas and of the absorbed gas separately. As for the local emission of radiative energy from turbulent combustion gas, the temporal mean value of the instantaneous emission energy was emitted steadily. As for the absorption, we evaluated the temporal mean amount of absorption by using the temporal mean value of instantaneous absorptance. Furthermore, the abovementioned emission energy and amount of absorption were evaluated for every elementary gray gas in the narrow band. Here, when the calculation of radiative heat transfer is performed, before performing the above calculation, we should individually evaluate the spatial distributions of the temporal mean value of instantaneous emission energy and instantaneous absorptance only once. By doing so, the calculation of radiative heat transfer in the area where the time-averaged distributions of temperature are given can be finished by calculating a single time step. Therefore, the calculation load will be reduced substantially. However, we still require several repeated calculations of convective heat transfer and of radiative heat transfer before the convergent result is acquired in cases where convection and radiation are combined. Note that when the assumption that temperature fluctuations of two arbitrary positions are independent from each other is made, it is not necessary to give a waveform of the temperature fluctuation history at each cell (discrete point) in a large-scale furnace. When a temperature fluctuation is given in a PDF statistically, the temporal mean value of instantaneous emission energy and of instantaneous absorptance can be evaluated separately.

As with our previous study targeting hydrogen flame (Takeuchi and Okamoto, 2017), here we included a transport equation for the temperature fluctuation intensity in the time-averaged governing equation solver that is implemented in the turbulent model, and the PDF of temperature fluctuation was expressed by using the obtained time-averaged temperature and temperature fluctuation intensity. By combining this calculation technique for radiative heat transfer with calculations of flow, mixture, and combustion, we can evaluate the heat transfer including radiation taking TRI into consideration in large-scale industrial furnaces enveloping turbulent hydrocarbon diffusion flames with sufficient accuracy and a feasible calculation load.

We compare this with a case that does not consider TRI as practical calculation. In this case, we applied non-gray analysis with the abovementioned method of bandwidth division in the narrow band. However, because a turbulent fluctuation was not considered, the method for reducing the calculation load associated with the detailed trace of turbulent fluctuation was not applied. Therefore, we evaluated the emission energy and amount of absorption of every elementary gray gas in the narrow band on the basis of the time-averaged temperature of gas.

We refer to the case that considers TRI as "Turbulent case" and the case that does not consider TRI as "Steady case" from now on.

\section{Examination target and method 3.1 Examination target}

In this section, we investigate the influence of TRI for a large-scale furnace enveloping turbulent hydrocarbon diffusion flame. First, we describe the examination target. In their numerical analysis flow and combustion calculations based on time-averaged equations and the calculation of radiative heat transfer, Yoshimoto et al. (Yoshimoto et al., 1998) focused on a cylindrical furnace $1.0 \mathrm{~m}$ in diameter containing a coaxial turbulent diffusion flame to examine the influence of changing the handling of the non-gray characteristics of gas. Their results showed that, in contrast to gray analysis, non-gray analysis could predict a value close to the actual measurement for the heat flux distribution of a side 
wall of the furnace. According to the result of the non-gray analysis, radiative heat transfer occupied a little over $80 \%$ of the amount of heat transfer to the side wall. With reference to this work, in order to target a situation where the contribution of radiation is higher, we utilized a large-scale furnace twice the size of the one used by Yoshimoto et al. Figure 2 shows a schematic view of the large-scale combustion furnace we used, which is drawn sideways for convenience. This is a cylindrical furnace with a diameter of $2.0 \mathrm{~m}$ and height of $9.6 \mathrm{~m}$ installed vertically with its upper end opened to the atmosphere. A fuel nozzle is installed in the center of the furnace bottom. There is ventilation hole for combustion air installed around the fuel nozzle, and an axial symmetry turbulent diffusion flame is formed in the vertical upward direction. Fuel was the same propane used by Yoshimoto et al. We also set the same air ratio (2.0) and mean velocities of the propane and air jet $(54.0 \mathrm{~m} / \mathrm{s}$ and $3.8 \mathrm{~m} / \mathrm{s}$, respectively) as Yoshimoto et al. The supply temperatures of the propane and combustion air were kept constant at $298.15 \mathrm{~K}$.

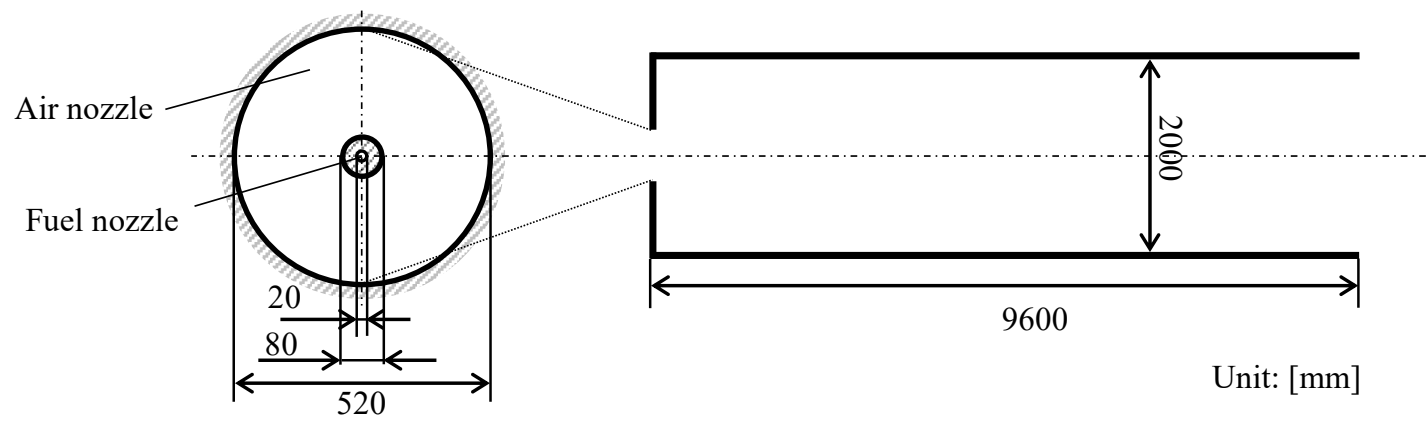

Fig.2 Schematic view of large-scale furnace utilized in this study.

\subsection{Calculation methods of flow, mixture, and combustion}

The same as in our previous report (Takeuchi and Okamoto, 2017), we assumed that the formed turbulent flame has an axial symmetry, and a two-dimensional cylindrical coordinates system was adopted. The spatial distributions of the time-averaged values of the temperature, gas composition, and so on in the furnace were acquired by the method for solving the time-averaged governing equation, which applied the $k-\varepsilon$ two equations model (Launder and Spalding, 1972) as a turbulent model (RANS). In addition, DOM, which is applied to the calculation method of radiative heat transfer explained in the previous section, was coupled to this, and the numerical prediction of the radiative heat transfer in the furnace was performed. The coupling was achieved by including the net emission energy evaluated by calculating the radiative heat transfer in the generation term of the transport equation of an enthalpy. The gas governing equation is

$$
\frac{\partial}{\partial t}(\rho \phi)+\frac{1 \partial}{r \partial r}(\rho r \nu \phi)+\frac{\partial}{\partial z}(\rho u \phi)=\frac{1}{r} \frac{\partial}{\partial r}\left(r \Gamma_{\phi} \frac{\partial \phi}{\partial r}\right)+\frac{\partial}{\partial z}\left(\Gamma_{\phi} \frac{\partial \phi}{\partial z}\right)+S_{\phi}
$$

where $\phi$ is a dependent variable expressed as $u$ for axial velocity, $v$ for radial velocity, $k$ for turbulent energy, $\varepsilon$ for dissipation rate of turbulent energy, $h$ for specific enthalpy, $m_{j}$ for mass fraction of chemical species $j$, and $Y$ for mixture fraction. $\Gamma_{\phi}$ and $S_{\phi}$ are the turbulent diffusion coefficients and source terms corresponding to the dependent variable $\phi$, respectively. The above values are listed in Table 1 . In both the equation and the table, $z$ and $r$ are coordinates of the axial direction and the radial direction, respectively. $P$ indicates pressure, $T$ indicates temperature, $\rho$ indicates density, and $R_{j}$ indicates generation rate of the chemical species $j$ by a chemical reaction. $Q_{R V}$ is the net radiative emission per unit time and unit volume. In addition, since the enthalpy of formation that is equivalent to the energy of a chemical bond is contained in the definition of specific enthalpy $h$, heat by combustion does not appear in the source term of the transport equation of enthalpy. Moreover, $\mu_{e f f}$ is a turbulent effective viscosity that is the sum of the laminar viscosity $\mu_{l}$ and the turbulent viscosity $\mu_{t}$ calculated by $\mu_{t}=C_{D} \times \rho k^{2} / \varepsilon$. The laminar viscosity $\mu_{l j}$ of the chemical species $j$ was evaluated using Sutherland's equations (Sutherland, 1895), and the laminar viscosity $\mu_{l}$ of the mixture gas was calculated using Sutherland-Wassiljewa's equation (Wassiljewa, 1904). $\sigma_{k}, \sigma_{\varepsilon}, \sigma_{h}$, and $\sigma_{Y}$ are an effective Prandtl number or an effective Schmidt number; the values are listed in Table 2. 
Takeuchi, Asao and Yamakawa, Journal of Thermal Science and Technology, Vol.16, No.2 (2021)

Table 1 Variables, source terms, and turbulent diffusion coefficients for gas governing equations.

\begin{tabular}{|cccl|}
\hline & $\phi$ & $\Gamma_{\phi}$ & $S_{\phi}$ \\
\hline Mass & 1 & 0 & 0 \\
\hline Axial momentum & $u$ & $\mu_{\text {eff }}$ & $\frac{\partial}{\partial z}\left(\mu_{\text {eff }} \frac{\partial \boldsymbol{u}}{\partial z}\right)+\frac{1}{r} \frac{\partial}{\partial r}\left(r \mu_{\text {eff }} \frac{\partial v}{\partial z}\right)-\frac{\partial \boldsymbol{P}}{\partial z}$ \\
\hline Radial momentum & $v$ & $\mu_{\text {eff }}$ & $\frac{\partial}{\partial z}\left(\mu_{\text {eff }} \frac{\partial \boldsymbol{u}}{\partial \boldsymbol{r}}\right)+\frac{1}{r} \frac{\partial}{\partial r}\left(r \mu_{\text {eff }} \frac{\partial v}{\partial r}\right)-2 \mu_{\text {eff }} \frac{v}{r^{2}}-\frac{\partial \boldsymbol{P}}{\partial r}$ \\
\hline Turbulent kinetic energy & $k$ & $\mu_{\text {eff }} / \sigma_{k}$ & $G_{k}-\rho \varepsilon$ \\
\hline Eddy dissipation rate & $\varepsilon$ & $\mu_{\text {eff }} / \sigma_{\varepsilon}$ & $\varepsilon\left(C_{\varepsilon 1} G_{k}-C_{\varepsilon 2} \rho \varepsilon\right) / k$ \\
\hline Mass fraction & $m_{i}$ & $\mu_{e f f} / \sigma_{m}$ & $R_{i}$ \\
\hline Mixture fraction & $Y$ & $\mu_{\text {eff }} / \sigma_{Y}$ & 0 \\
\hline Enthalpy & $h$ & $\mu_{\text {eff }} / \sigma_{h}$ & $-Q_{R V}$ \\
\hline
\end{tabular}

Table 2 Parameters for $k-\varepsilon$ model.

\begin{tabular}{|c|c|c|c|c|c|c|}
\hline$C_{\varepsilon 1}$ & $C_{\varepsilon 2}$ & $\sigma_{k}$ & $\sigma_{\varepsilon}$ & $\sigma_{m}$ & $\sigma_{Y}$ & $\sigma_{h}$ \\
\hline 1.44 & 1.92 & 1.0 & 1.3 & 0.7 & 0.7 & 0.7 \\
\hline
\end{tabular}

The gas compositions taken into consideration were $\mathrm{C}_{3} \mathrm{H}_{8}, \mathrm{O}_{2}, \mathrm{~N}_{2}, \mathrm{CO}_{2}$, and $\mathrm{H}_{2} \mathrm{O}$ as a reactant and product in the combustion reaction of propane and air. The one-step overall irreversible reaction of propane was assumed as a combustion reaction mechanism and Magnussen's eddy dissipation model (Magnussen and Hjertager, 1976) was used as a turbulent combustion model. Here, the value of the experimental constant $B$ of Magnussen's model was set to 4.0, the same as the reference. The time-averaged temperature of gas was acquired by

$$
h=\sum_{j}\left(m_{j} \int_{T_{0}}^{\bar{T}} c_{P_{j}} d T\right)+\sum_{j} m_{j} h_{0_{j}}
$$

where $T_{0}$ is base temperature $(=298.15 \mathrm{~K}), c_{P j}$ is specific heat at constant pressure of $j$ component, and $h_{0 j}$ is the standard enthalpy of the formation of $j$ component.

Next, as described in section 2, when calculating the radiative heat transfer taking TRI into consideration (Turbulent case), we included the transport equation for the temperature fluctuation intensity in the above time-averaged governing equation solver, and the PDF of the temperature fluctuation was expressed by using the obtained time-averaged temperature and temperature fluctuation intensity. The method is described below.

First, for the local temperature fluctuation intensity, the fluctuation intensity $g_{T}$ of temperature $T(t)$ (the secondary moment around the averaged value) was defined by Eq. (4), and the spatial distribution of $g_{T}$ was obtained using the transport equation (5).

$$
\begin{aligned}
& g_{T}=\frac{1}{t} \int_{0}^{t}\left\{T\left(t^{\prime}\right)-\bar{T}\right\}^{2} d t^{\prime} \\
& \frac{1}{r} \frac{\partial}{\partial r}\left(\rho r v g_{T}\right)+\frac{\partial}{\partial z}\left(\rho u g_{T}\right)=\frac{\partial}{\partial z}\left(\frac{\mu_{e f f}}{\sigma_{g_{T}}} \frac{\partial g_{T}}{\partial z}\right)+\frac{1}{r} \frac{\partial}{\partial r}\left(r \frac{\mu_{e f f}}{\sigma_{g_{T}}} \frac{\partial g_{T}}{\partial z}\right)+C_{g_{1}} \mu_{e f f}\left[\left(\frac{\partial \bar{T}}{\partial z}\right)^{2}+\left(\frac{\partial \bar{T}}{\partial r}\right)^{2}\right]-C_{g 2} \rho g_{T} \frac{\varepsilon}{k} .
\end{aligned}
$$

Here, $\sigma_{g T}$ is an effective Schmidt number to $g_{T}$, and its value is 0.7. $C_{g 1}$ and $C g_{2}$ are model constants with values of 2.8 and 1.92, respectively (Khalil et al., 1975).

Next, we assumed that the probability of a certain instantaneous temperature $T(t)$ follows a Gaussian distribution and obtained the PDF of local temperature fluctuation in the furnace by using the spatial distributions of time-averaged temperature $T$ and standard deviation of temperature fluctuation $s_{T}$ (square root of $g_{T}$ ). This PDF was created as a 
clipped-Gaussian PDF (clipped temperature; bottom: $\bar{T}-3 s_{T}$ or the supplied temperature of fuel and air (298.15 K), top: $T+3 s_{T}$ or the adiabatic combustion temperature of propane $(2268 \mathrm{~K})$ ). The clipped-Gaussian PDF was divided into 200 steps of temperature, and temporal mean values of instantaneous emission energy and instantaneous absorptance were evaluated for every elementary gray gas in the narrow band based on the appearance probability of each step of temperature. Further, when performing a clipping at the temperature $\left(\bar{T} \pm 3 s_{T}\right)$, the probability that the temperature outside this range will appear is below $0.3 \%$, so we presume that the influence on the evaluated value of emission energy and absorption is small. As for the step number of temperature, even if it was increased, we confirmed that the differences in the emission energy and absorption were small enough that they can be ignored.

\subsection{Analysis of radiative heat transfer}

We used DOM in a three-dimensional cylindrical coordinates system (Carlson and Lathrop, 1968; Jamaluddin and Smith, 1988) for the analysis of radiative heat transfer. The radiation transport equation for dealing with axial symmetry in a three-dimensional cylindrical coordinates system is

$$
\frac{\mu_{D}}{r} \frac{\partial(r I)}{\partial r}+\zeta_{D} \frac{\partial I}{\partial z}-\frac{1}{r} \frac{\partial\left(\eta_{D} I\right)}{\partial \omega_{D}}=-K I+K I_{B}
$$

where $I$ is the radiative intensity, $I_{B}$ is the radiative intensity of a black body, and $K$ is the absorption coefficient. $\mu_{D}$, $\eta_{D}$, and $\zeta_{D}$ are the direction cosines to the radial direction, the circumferential direction, and the axial direction axis, respectively, and $\omega_{D}$ is an azimuth. Generally, although the entire solid angles $4 \pi$ are discretized in the $S(S+2)$ directions $(S=2,4,6, \ldots)$ in DOM, we adopted the S6 method in this study. In addition, as a result of preliminary examination, we confirmed that the result of radiative heat transfer at the furnace side wall calculated by the S6 method was almost the same as that calculated by the S8 method, which has a higher order.

In this study, since we perform a calculation of radiative heat transfer for every 24 elementary gray gases contained in the narrow band $\left(100 \mathrm{~cm}^{-1}\right.$ wide $)$, the directional discretized radiation transport equation is expressed as

$$
\frac{\mu_{D}{ }^{m}}{r} \frac{\partial\left(r I_{\eta(n b), i}^{m}\right)}{\partial r}+\zeta_{D}{ }^{m} \frac{\partial I_{\eta(n b), i}^{m}}{\partial z}-\frac{1}{r} \frac{\partial\left(\eta_{D}{ }^{m} I_{\eta(n b), i}^{m}\right)}{\partial \omega_{D}}=-I_{A, \eta(n b), i}^{m}+w_{t}^{m} I_{E, \eta(n b), i}
$$

where subscript $\eta_{(n b), i}$ refers to an $i$-th elementary gray gas in the $n b$-th narrow band, superscript $m$ is a number of the discretized direction, and $w_{t}^{m}$ is a weight coefficient of discretization of the $m$ direction. The direction cosines and weight coefficients were determined using Fiveland's method (Fiveland, 1988). $I_{E, \eta(n b), i}$ is the directional emissive power from the $i$-th elementary gray gas in the $n b$-th narrow band, and $I_{A, \eta(n b), i}^{m}$ is the value absorbed by the $i$-th elementary gray gas in the $n b$-th narrow band. Note that the evaluation methods used for these are different in the Turbulent case and Steady case.

In the Turbulent case, $I_{E, \eta(n b), i}$ and $I_{A, \eta(n b), i}^{m}$ were evaluated by using the temporal mean value of instantaneous emission energy and the instantaneous absorptance, respectively, acquired by

$$
\begin{aligned}
& \bar{I}_{E, \eta(n b), i}=\sum_{N=1}^{200}\left[\langle K\rangle_{\eta(n b), i}\left(T_{N}\right) \cdot\langle I\rangle_{B, \eta(n b)}\left(T_{N}\right) \cdot F\left(T_{N}\right)\right], \\
& \bar{I}_{A, \eta(n b), i}^{m}=\sum_{N=1}^{200}\left[\langle K\rangle_{\eta(n b), i}\left(T_{N}\right) \cdot F\left(T_{N}\right)\right] \cdot I_{\eta(n b), i}^{m} .
\end{aligned}
$$

Here, $\left\langle K>_{\eta(n b), i}\left(T_{N}\right)\right.$ is the absorption coefficient of the $i$-th elementary gray gas in the $n b$-th narrow band evaluated at temperature $T_{N}$, and it is evaluated as the arithmetic average of the absorption coefficient in the bandwidth allocated with Eq. (1). Further, $\langle I\rangle_{B, \eta(n b)}\left(T_{N}\right)$ is the value that equalizes the spectral radiative intensity of the black body based on temperature $T_{N}$ in the bandwidth that the $n b$-th narrow band covers. $F\left(T_{N}\right)$ is an appearance probability of temperature 
$T_{N}$, and it was obtained by the method explained in Section 3.2.

In the Steady case, $I_{E, \eta(n b), i}$ and $I_{A, \eta(n b), i}$ were evaluated on the basis of time-averaged temperature $T$, acquired by

$$
\begin{aligned}
& I_{E, \eta(n b), i}=\langle K\rangle_{\eta(n b), i}(\bar{T}) \cdot\langle I\rangle_{B, \eta(n b)}(\bar{T}), \\
& I_{A, \eta(n b), i}^{m}=\langle K\rangle_{\eta(n b), i}(\bar{T}) \cdot I_{\eta(n b), i}^{m} .
\end{aligned}
$$

Further, when the spectrum of the absorption coefficient was calculated, the method of relating the temperature to the partial pressures of gases shown in Fig. 1 was used. In that case, according to the value of mixture fraction $Y$, when the mixture ratio of the fuel and the air was beyond a stoichiometric ratio, it was classified as the inside of the flame, and when it was below that, it was classified as the outside of the flame. The appropriate method to acquire the spectrum of the absorption coefficient inside or outside the flame was then used.

\subsection{Analysis conditions and solutions}

We used the same calculation grid in this study as our previous report (Takeuchi and Okamoto, 2017), as shown in Fig. 3. The computational domain was divided into 267 divisions axially and 69 divisions radially, with the divisions placed at uneven intervals so that the neighborhood of the fuel nozzle could be divided more finely. The same division method was used for the calculation of radiative heat transfer, although it was not divided in the direction of the circumference since it had an axial symmetry.

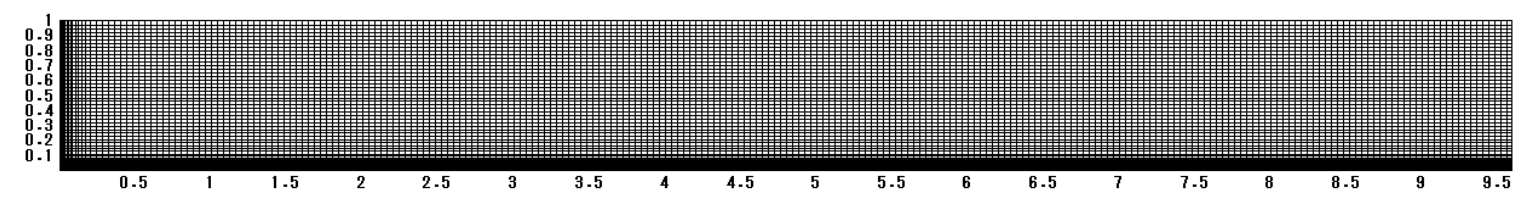

Fig.3 Schematic view of calculation grid used in this study.

The discretization method of the equations and the solution were also the same as the previous report. The transport equations (2) were discretized using a finite volume method, and the Tri-Diagonal-Matrix Algorithm (TDMA) was used for the solution of the discretized equations. We used the Semi Implicit Method for Pressure Linked Equations Revised (SIMPLER) (Patankar and Spalding, 1970) for the coupling of the velocity field and the pressure field.

The setting method for the boundary conditions was basically the same as the previous report. The boundary conditions are listed in Table 3 and explained as follows.

For the boundary conditions of the entrances of fuel and combustion air, the radial velocity $v$ was set to $0 \mathrm{~m} / \mathrm{s}$. The axial velocity $u$ of the combustion air was set constant at $3.8 \mathrm{~m} / \mathrm{s}$ with a flat distribution, and that of the fuel was set to the distribution obtained by calculating the developed flow in a nozzle using the $k-\varepsilon$ model. The average value of the axial velocity of the fuel was $54.0 \mathrm{~m} / \mathrm{s}$. The turbulent energy $k$ of the fuel was set to the distribution obtained by calculating using the $k-\varepsilon$ model, the same as $u$. The turbulent energy of the combustion air was given as a flat distribution assuming the relative turbulence to air flow velocity, $3.8 \mathrm{~m} / \mathrm{s}$, to be $30 \%$, the same as Yoshimoto et al. The dissipation rate $\varepsilon$ of the turbulent energy of fuel was given by $\varepsilon=0.3 k(\partial u / \partial r)$ as conditions that the generation and dispersion of turbulent energy keep a local equilibrium. For the dissipation rate $\varepsilon$ of the turbulent energy of combustion air, the characteristic length of turbulence was assumed to be 0.01 times the length of an air nozzle radius and was given a constant value $\varepsilon=34.62 \mathrm{k}^{2 / 3}$. Specific enthalpy $h$ was evaluated from the supply temperature of fuel and air as $298.15 \mathrm{~K}$, and it included the standard enthalpy of formation. The temperature fluctuation intensity $g_{T}$ was set to 0 .

As for the boundary conditions of the outflow at the top of the furnace, since the downstream outflow boundary was exposed to atmospheric pressure, the gauge pressure was set to $0 \mathrm{kPa}$. The radial velocity $v$ was also set to $0 \mathrm{~m} / \mathrm{s}$. For the other dependent variables, we applied the condition that the gradient of an axial direction was $0(\partial \phi / \partial z=0)$.

For the boundary conditions of the central axis, since axial symmetry was assumed, we applied the condition that the gradient of a radial direction was $0(\partial \phi / \partial r=0)$ to all the dependent variables (other than radial velocity $v)$ on a central axis boundary. The radial velocity $v$ was set to $0 \mathrm{~m} / \mathrm{s}$. 
For the boundary conditions of the furnace wall, we set the conditions that the gradient of a radial direction was 0 $(\partial \phi / \partial r=0)$ for the mass fraction $m_{j}$ of chemical species, mixture fraction $Y$, and the temperature fluctuation intensity $g_{T}$. The radial velocity $v$ was set to $0 \mathrm{~m} / \mathrm{s}$. Wall functions were used for the axial velocity $u$, turbulent energy $k$, and dispersion rate $\varepsilon$. The wall function was also applied for convective heat transfer to the furnace wall (Launder and Spalding, 1974).

Table 3 Boundary conditions.

\begin{tabular}{|l|l|}
\hline Center axis & Symmetric \\
\hline \multirow{3}{*}{ Fuel inlet } & $\begin{array}{l}v=0, u=54.0 \mathrm{~m} / \mathrm{s}, \bar{k}=393 \mathrm{~m}^{2} / \mathrm{s}^{2}, \varepsilon=0.3 k(\partial u / \partial r), \quad m_{\mathrm{C} 3 \mathrm{H} 8}=1, Y=1, h=-2.36 \mathrm{MJ}, \\
\end{array}$ \\
\hline \multirow{2}{*}{ Air inlet } & $\begin{array}{l}v=0, u=3.8 \mathrm{~m} / \mathrm{s}, k=1.95 \mathrm{~m}^{2} / \mathrm{s}^{2}, \varepsilon=34.62 k^{2 / 3}, m_{\mathrm{C} 3 \mathrm{H} 8}=0, m_{\mathrm{O} 2}=0.232, \\
\end{array}$ \\
\hline Bottom of furnace & $v=0\left(\phi=Y, h, g_{T}\right)$ \\
\hline Outlet of furnace & $v=0, \partial \phi / \partial z=0\left(\phi=u, k, \varepsilon, m, Y, h, g_{T}\right)$ \\
\hline Side wall of furnace & $v=0$, wall function $(\phi=u, k, \varepsilon, h), \partial \phi / \partial r=0\left(\phi=m, Y, g_{T}\right)$ \\
\hline
\end{tabular}

\section{Results and discussion}

\subsection{Effects of TRI on radiative heat transfer arriving at side wall of the combustion furnace}

First, we examined the effects of TRI on radiative heat transfer arriving at the side wall of the combustion furnace. Figure 4 shows the distributions of radiative heat flux $q_{R}$ and convective heat flux $q_{T}$ at the side wall of the furnace. The radiative heat flux $q_{R}$ is a result from all wave number regions $(0-10000 \mathrm{~cm}-1)$ obtained by integrating the respective results after performing calculations for every elementary gray gas in each narrow band. In the figure, blue and red lines respectively indicate the Turbulent case (that considers TRI) and the Steady case (that does not consider TRI). Solid lines indicate the results of $q_{R}$ and broken lines indicate the results of $q_{T}$. Since the convective heat flux $q_{T}$ is smaller than the radiative heat flux $q_{R}$, it has been moved to the scale of the right axis. Table 4 shows each total amount of the radiative heat transfer and convective heat transfer, which were obtained by multiplying each of $q_{R}$ and $q_{T}$ by a heating surface area. The difference by not considering TRI is also shown in the Steady case column.

First, in the large-scale combustion furnace with the $1 \mathrm{~m}$ radius, we found that the radiative heat transfer occupied about $87 \%$ of the total amount of the heat transferred to the side wall by radiation and convection. In addition, it turned out to be the area with a very high importance for the radiative heat transfer. On the basis of this finding, when two distributions of radiative heat flux $q_{R}$ were compared, two cases showed distributions of monotonous convexity that possessed a peak value around the center of the furnace. We also found that the influence of the predicted position where radiative heat flux shows the maximum was shifted to the downstream side a little in the Steady case that doesn't consider TRI. It seems here that a different prediction of the suitable arrangement for a heated product may be generated in the present practical calculation based on time-averaged value. In addition, compared to the Turbulent case, the radiative heat flux in Steady case was underestimated in the upstream area to $z=4.9 \mathrm{~m}$, and conversely, it was overestimated in the downstream area. When the total amount of the radiative heat transfer at the furnace side wall (see Table 4) appeared, it turned out that the total amount of radiative heat transfer in the Steady case was underestimated compared with in the Turbulent case. If the calculation of radiative heat transfer is performed while disregarding turbulent fluctuation, the amount of radiative energy at the furnace wall is underestimated. This insight was also evident in a previous report (Takeuchi et.al, 2013) that examined one typical optical path in the furnace enveloping the hydrogen flame and in another report (Takeuchi and Okamoto, 2020) that examined one typical optical path in the furnace enveloping the hydrocarbon flame. We can conclude that this same finding will be true even if the whole furnace is examined. However, although the relative difference caused by disregarding turbulent fluctuation was about $-30 \%$ in the previous report (Takeuchi and Okamoto, 2020), in our study we found that the relative difference in the whole furnace was $-3.65 \%$, which is relatively small compared to the difference in the previous report. We conclude that underestimating the radiative heat flux at an upstream region limits how much the gas temperature falls and results in an overestimation of the emission energy at the downstream region.

In contrast, when we take a look at the convective heat flux $q_{T}$, the difference caused by not considering TRI appeared only at the downstream region, and the total amount of convective heat transfer in the Steady case was 
overestimated compared with in the Turbulent case. As a result, in terms of the total amount of heat transferred to the side wall by radiation and convection obtained by adding $Q_{R}$ and $Q_{T}$, the underestimation by not considering TRI was mitigated a little.

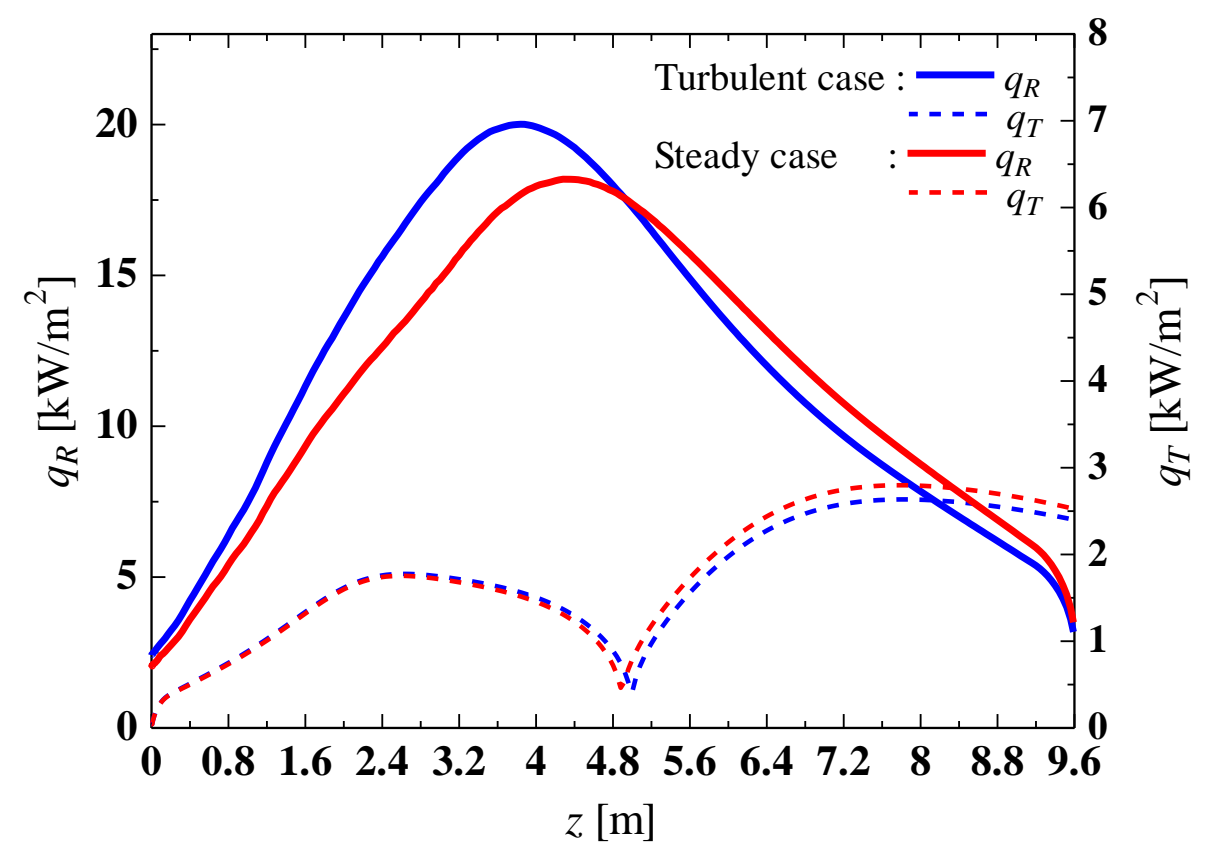

Fig.4 Effects of TRI on radiative and convective heat flux to the side wall of furnace.

Table 4 Effects of TRI on total amount of heat transferred to the side wall by radiation and convection.

\begin{tabular}{|c|c|c|}
\hline & Turbulent case & Steady case [ difference ( relative difference) ] \\
\hline$Q_{R}$ & $726.23 \mathrm{~kW}$ & $692.19 \mathrm{~kW}[-34.04 \mathrm{~kW}(-4.69 \%)]$ \\
\hline$Q_{T}$ & $104.94 \mathrm{~kW}$ & $108.63 \mathrm{~kW}[+3.69 \mathrm{~kW}(3.52 \%)]$ \\
\hline$Q_{R}+Q_{T}$ & $831.17 \mathrm{~kW}$ & $800.82 \mathrm{~kW}[-30.35 \mathrm{~kW}(-3.65 \%)]$ \\
\hline
\end{tabular}

\subsection{Effects of TRI on temperature in the furnace}

Even if TRI was not considered, there was no great difference in the evaluated total amount of heat transfer. We presume that this stems from the fact that the distribution of temperature in a large-scale furnace was different, as discussed in the previous section. To examine this is more detail, we next compared the distribution of temperature in the furnace.

Figure 5 shows the time-averaged temperature and the temperature fluctuation intensity in the furnace as a distribution diagram with gradation. Four typical cross-sections from the diagram of time-averaged temperature are shown in Fig. 6, with the radial distribution of the time-averaged temperature removed. Even if TRI was not considered at an upstream region, there was almost no influence on the temperature, but we found that the temperature was evaluated highly at a downstream region.

Figure 7 shows the net radiative emission $Q_{R V}$ at the same point on each cross section obtained by taking the difference between the emission energy per unit volume and the amount of absorption absorbed at that point. The maximum of the net radiative emission at each cross section tended to be overestimated in the Steady case. However, in the area outside of the flame with the big percentage that occupies space, when TRI was not considered, we found that the net radiative emission was underestimated. When we compared it with the distribution diagram of the temperature fluctuation intensity from Fig. 5, the overall differences at the $z=2.0 \mathrm{~m}$ cross section and the $z=3.0 \mathrm{~m}$ cross section with the strong temperature fluctuation intensity were remarkable. We conclude that the nonlinear temperature dependency of the emission makes a significant contribution. When TRI was not considered, we found that the amount to which the temperature gas fell was estimated as low to underestimate the radiative emission. The difference in the radiative emission appeared at the $z=1.0 \mathrm{~m}$ cross section located in an upstream region, but this same temperature 
difference was not evidenced at the same cross section located in the upstream region. This is presumably because, for the temperature difference between two cases to appear cumulatively along a streamline, the temperature difference could not easily appear in the upstream flame region. Furthermore, since the flow velocity was large and the transportation of the thermal energy by the convection was significant in the upstream region, we speculate that this is the reason the effect of the thermal energy transportation by radiation became relatively small. From the above, in the Steady case that doesn't consider TRI, we conclude that an underestimation of the radiative emission leads to an underestimation of the radiative heat flux arriving at the side wall of the combustion furnace in the upstream region, and an overestimation of the temperature leads to an overestimation of the radiative heat flux in the downstream region. As a result, even if TRI is not considered, the underestimation and overestimation of radiative heat flux cancel each other out, so we assume that the total amount of radiative heat transfer arriving at the side wall of the large-scale combustion furnace does not make any large difference.

We compared the above results obtained for the hydrocarbon flame with those obtained for the hydrogen flame in our previous report (Takeuchi and Okamoto, 2017) and found that, because the temperature level was different for the hydrogen flame in propane, there were differences in the total amount of heat transferred to the side wall by radiation and convection, but the influential behavior of TRI was almost similar. When the kind of gaseous fuel is different, the degree of influence of TRI is also different. Even so, there is a possibility that the kind of gaseous fuel does not affect the influential behavior of TRI.

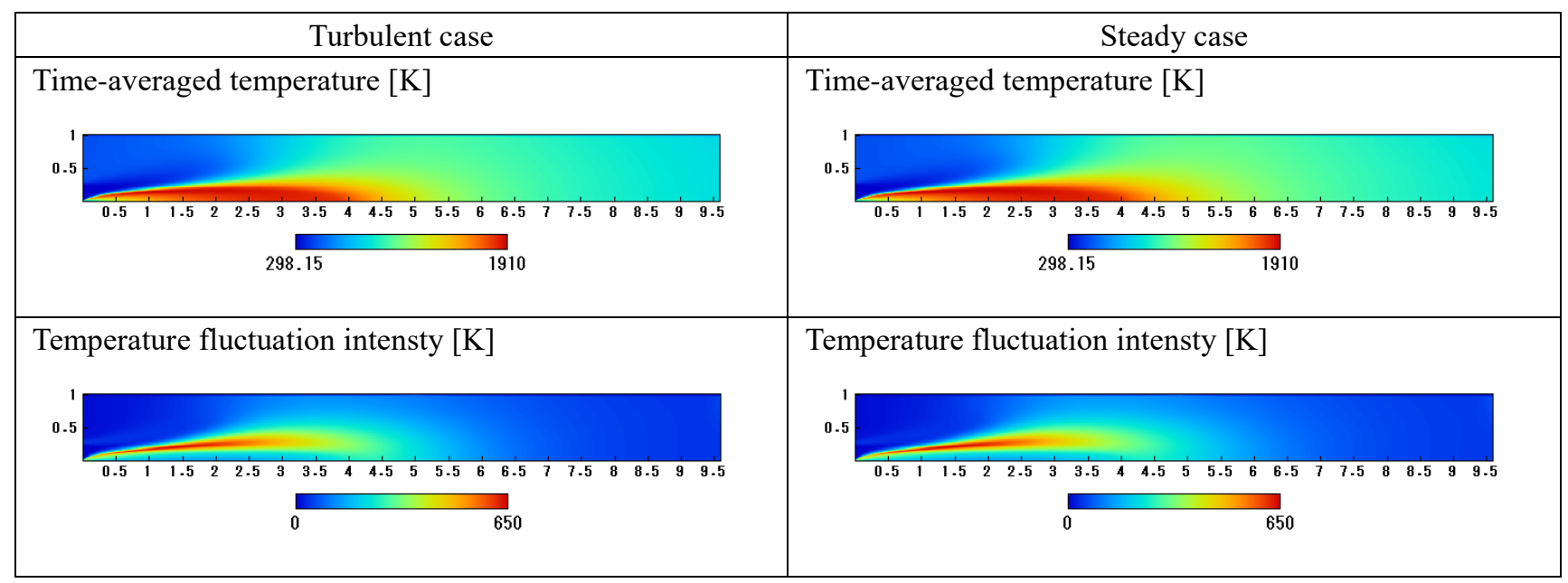

Fig.5 Effects of TRI on the spatial distributions of time-averaged temperature and temperature fluctuation intensity.

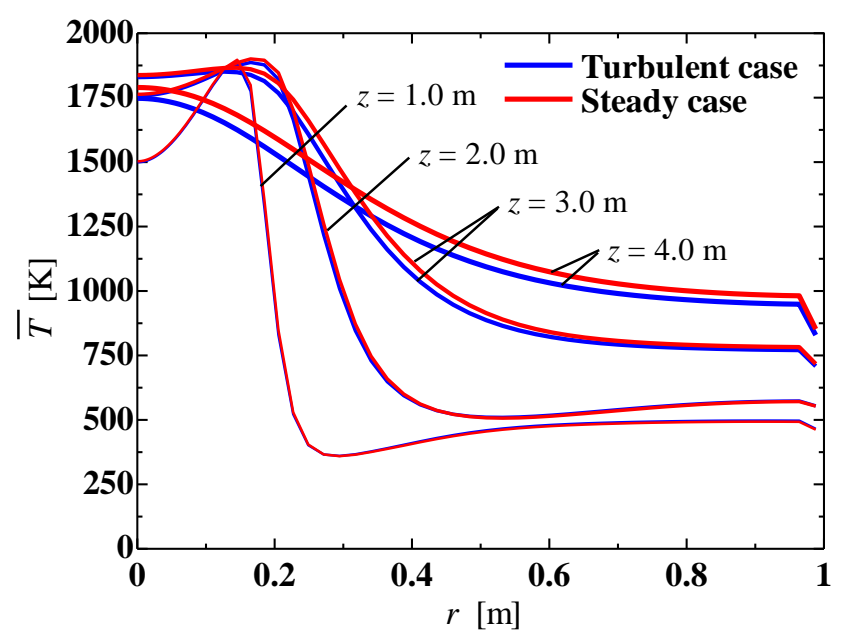

Fig.6 Effects of TRI on the spatial distributions of time-averaged temperature.

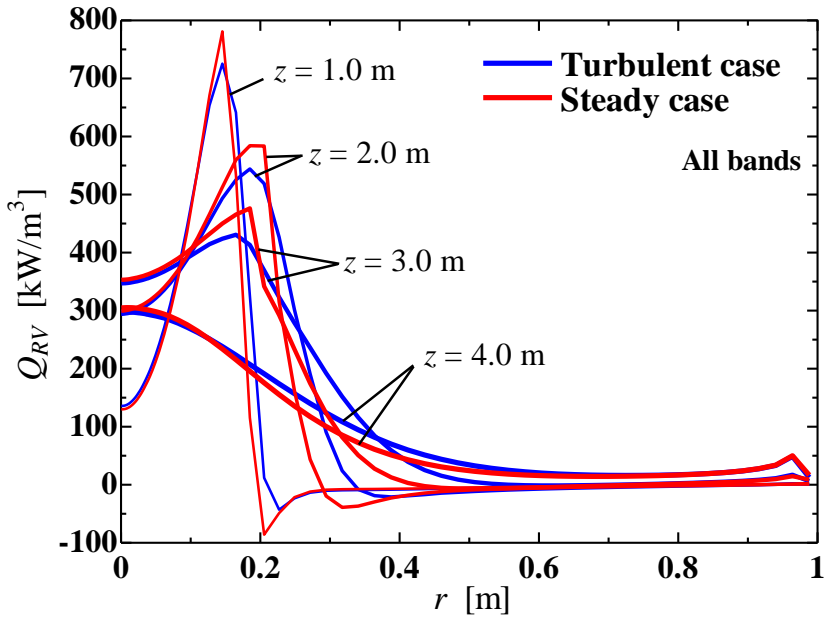

Fig.7 Effects of TRI on the spatial distributions of the net radiative emission. 


\subsection{Effects of TRI on the gas composition}

In the previous section, we showed that TRI has an influence on the prediction of temperature distribution in the large-scale furnace. There is a possibility that TRI might also have an influence on the prediction of gas composition, so we examined the influences of TRI on the gas composition next.

Figure 8 show the radial distributions of the molarities of chemical species at the sections of $z=2 \mathrm{~m}$ and $z=4 \mathrm{~m}$, where solid and broken lines indicate the results of the Turbulent and Steady cases, respectively. As we can see, a difference in temperature distribution barely appeared in the distributions of chemical species, and almost the same distributions were obtained even if TRI was not considered. This tendency was also reported in a previous study (Takeuchi and Okamoto, 2017). As described in the previous report, in terms of the substance for which the generation rate depends on temperature sensitively (e.g., NOx), there is a possibility that a large error will appear in the predicted value of the generation amount.
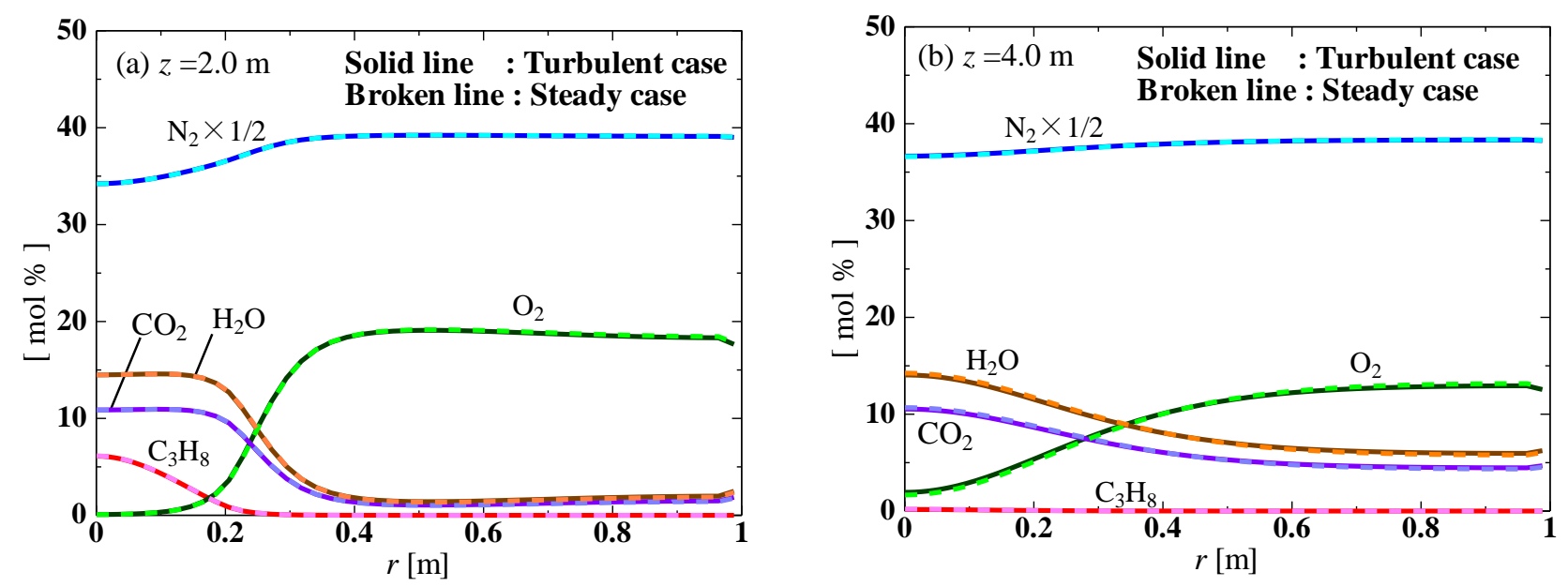

Fig.8 Effects of TRI on the spatial distributions of chemical species at (a) $z=2.0 \mathrm{~m}$ and (b) $z=4.0 \mathrm{~m}$.

\section{Conclusion}

In this study, we focused on a large-scale industrial furnace enveloping a turbulent hydrocarbon diffusion flame to examine the influence of TRI on the radiative heat transfer arriving at the side wall of the furnace. Our findings are summarized as follows.

1. In the calculation of radiative heat transfer combined with the calculations of gas flow, mixture, and combustion, if TRI is not considered, the predicted total amount of the heat transferred to the side wall by radiation and convection will be underestimated. However, the degree of difference is relatively small. At the same time, disregarding TRI causes the predicted position at which the radiative heat flux shows the maximum to shift a little to the downstream side. In practical calculation based on the time-averaged value, there is a possibility that a difference in terms of the suitable arrangement of a heated product will be generated. As such, the above influence cannot be ignored.

2. If TRI is not considered, in order to underestimate the emission of radiative energy, the temperature fall of the flame is estimated to be low. Although the influence that underestimates the temperature fall of the flame barely appears in the upstream region, it is quite obvious in the downstream region and causes overestimation of the temperature in the downstream region of the furnace. As a result, the evaluated radiative heat flux to the furnace wall is underestimated in the upstream region, and is overestimated in the downstream region. The underestimation and overestimation of radiative heat flux cancel each other out, so a large difference does not appear in the total amount of the radiative heat transfer arriving at the side wall of the furnace. 
3. The difference barely appears in the distributions of chemical species even if the TRI is not considered.

4. The following are assumed from comparison of the results obtained for the hydrocarbon flame and those obtained for the hydrogen flame in our previous report. The difference in the total amount of heat transferred to the side wall by radiation and convection appears because the temperature of a flame is different when different kinds of gaseous fuel are used. Therefore, the degree of influence of TRI is different. Even so, there is a possibility that the kind of gaseous fuel does not affect the influential behavior of TRI.

\section{References}

Chandy, A. J., Glaze, D. J. and Frankel, S. H., A hybrid large eddy simulation/filtered mass density function for the calculation of strongly radiating turbulent flames, ASME Journal of Heat Transfer, Vol.131, No.5 (2009), 051201.1-051201.9.

Carlson, B. G. and Lathrop, K. D., Transport theory -The method of discrete ordinates, Computing Methods in Reactor Physics, H.Greenspan ed., Gordon and Breach Science Public, New York, U.S.A. (1968), pp.171-266.

Coelho, P. J., Approximate solutions of the filtered radiative transfer equation in large eddy simulations of turbulent reactive flows, Combustion and Flame, Vol.156, No.5 (2009), pp.1099-1110.

Coelho, P. J., Teerling, O. J. and Roekaerts, D., Spectral radiative effects and turbulence / radiation interaction in a non-luminous turbulent diffusion flame, Combustion and Flame, Vol. 133, No.1-2 (2003), pp.75-91.

Edwards, D.K., Advances in Heat Transfer, Academic Press, Vol.12 (1976), pp.115-193.

Fiveland, W. A., Three-dimensional radiative heat-transfer solutions by the discrete-ordinates method, Journal of Thermophysics, 2 (1988), pp.309-316.

Gupta, A., Haworth, D. C. and Modest, M. F., Turbulence-radiation interactions in large-eddy simulations of luminous and nonluminous nonpremixed flames, Proceedings of the Combustion Institute, Vol.34, No.1 (2013), pp.1281-1288.

Gupta, A., Modest, M. F. and Haworth, D. C., Large-eddy simulation of turbulence-radiation interactions in a turbulent planar channel flow, ASME Journal of Heat Transfer, Vol.131, No.6 (2009), 061704.1-061704.8.

Habibi, A., Merci, D. and RoeKaerts, D., Turbulence radiation interaction in Reynolds-averaged Navier-Stokes simulations of nonpremixed piloted turbulent laboratory-scale flames, Combustion and Flame, Vol. 151, No.1-2 (2007), pp.303-320.

Jamaluddin, A. S. and Smith, P. J., Predicting radiative transfer in axisymmetric cylindrical enclosures using the discrete-ordinate method, Combustion Science and Technology, Vol.62 (1988), pp.173-186.

Khalil, E. E., Spalding, D. B. and Whitelaw, J. H., The calculation of local flow properties in two-dimensional furnaces, International Journal of Heat Mass Transfer, Vol.18 (1975), pp.775-792.

Launder, B. E. and Spalding, D. B., Mathematical models of turbulence, Academic Press (1972).

Launder, B.E. and Spalding, D. B., The numerical computation of turbulent flows, Computer Methods in Applied and Mechanics and Engineering, Vol.3 (1974), pp.269-289.

Li, G. and Modest, M. F., Importance of turbulence-radiation interactions in turbulent diffusion jet flames, ASME Journal of Heat Transfer, Vol.125, No.5 (2003), pp.831-838.

Magnussen, B. F. and Hjertager, B. H., On mathematical modeling of turbulent combustion with special emphasis on soot formation and combustion, 16th Symposium of International on Combustion (1976), pp.719-729.

Mazumder, S. and Modest, M. F., A probability density function approach to modeling turbulence-radiation interactions in nonluminous flames, International Journal of Heat and Mass Transfer, Vol.42, No.6 (1999), pp.971-991.

Modest, M. F. and Haworth, D. C., Radiative heat transfer in turbulent combustion systems -Theory and applications-, Springer Briefs in Applied Sciences and Technology (2016).

Patankar, S. V. and Spalding, D. B., Heat and mass transfer in boundary layers, Intertext Book (1970).

Poitou, D., Amaya, J., Hafi, M. E. and Cuenot, B., Analysis of the interaction between turbulent combustion and thermal radiation using unsteady coupled LES/DOM simulations, Combustion and Flame, Vol.159, No.4 (2012), pp.1605-1618.

Poitou, D., Hafi, M. E. and Cuenot, B., Analysis of radiation modeling for turbulent combustion: development of a 
methodology to couple turbulent combustion and radiative heat transfer in LES, ASME Journal of Heat Transfer, Vol. 133, No.6 (2011), 062701.1-062701.10.

Rejeb, S. B. and Echekki, T., Thermal radiation modeling using the LES-ODT framework for turbulent combustion flows, International Journal of Heat and Mass Transfer, Vol.104 (2017), pp.1300-1316.

Roger, M., Coelho, P.J. and da Silva, C.B., Relevance of the subgrid-scales for large eddy simulations of turbulence-radiation interactions in a turbulent plane jet, Journal of Quantitative Spectroscopy and Radiative Transfer, Vol.112 (2011), pp.1250-1256.

Roger, M., Da Silva, B. D. and Coelho, P. J., Analysis of the turbulence-radiation interactions for large eddy simulations of turbulent flows, International Journal of Heat and Mass Transfer, Vol.52, No. 9-10 (2009), pp.2243-2254.

Rothman, L.S., HITRAN database, Harvard Smithsonian Center, available from < http://www.cfa.harvard.edu / HITRAN/>, (accessed on 5 June, 2020).

Rothman, L.S., HITEMP DVD-ROM, Ontar Corporation (2010).

Somnath, G., Rainer, F. and Christian, S., Contrasting turbulence-radiation interaction in supersonic channel and pipe flow, International Journal of Heat and Fluid Flow, Vol.48 (2014), pp.24-34.

Sutherland, W., The Viscosity of Mixed Gases, Philosophical Magazine, Vol.40 (1895), pp.421-431.

Takeuchi, S. and Okamoto, T., An efficient method of non-gray analysis for studying radiative heat transfer in furnaces with comparable accuracy to line-by-line analysis (Proposal of method and examination in isothermal field), Transactions of the Japan Society of Mechanical Engineers (in Japanese), Series B,Vol.78, No.792 (2012a), pp.1409-1420.

Takeuchi, S. and Okamoto, T., An efficient method of non-gray analysis for studying radiative heat transfer in furnaces with comparable accuracy to line-by-line analysis (Proposal of method and examination in non-isothermal field), Transactions of the Japan Society of Mechanical Engineers (in Japanese), Series B,Vol.78, No.792 (2012b), pp.1421-1431.

Takeuchi, S. and Okamoto, T., Method for handling turbulent fluctuation in radiative heat transfer in furnaces (Proposal of efficient method and examination based on absorption line database), Transactions of the Japan Society of Mechanical Engineers (in Japanese), Series B,Vol.79, No.799 (2013), pp.452-464.

Takeuchi, S. and Okamoto, T., Influence of turbulence-radiation interaction on the radiative heat transfer to the wall of large-scale industrial furnaces and temperature distribution in the furnaces, Transactions of the JSME (in Japanese), Vol.83, No.852 (2017), DOI:10.1299/transjsme.17-00041.

Takeuchi, S. and Okamoto, T., Proposal of an efficient method for handling turbulent fluctuation on numerical simulations of radiative heat transfer in the large-scale industrial furnaces including hydrocarbon flame, Transactions of the JSME (in Japanese), Vol.86, No.883 (2020), DOI:10.1299/transjsme.19-00386.

Takeuchi, S., Konishi, T. and Okamoto, T., Fundamental examination about the influence of turbulent fluctuation on the radiative heat flux arriving at the wall of industrial furnaces, Transactions of the Japan Society of Mechanical Engineers, Series B,Vol.79, No.799 (2013), pp.439-451 (in Japanese).

TNF Workshop, Sandia/ TUD piloted CH4/Air jet flames, available from $<$ http://www.sandia.gov/TNF/DataArch /FlameD.html>, (accessed on 26 May, 2021).

Yoshimoto, T., Okamoto, T., Takagi, T. and Katsuki, M., Numerical simulation of combustion and nongray radiative heat transfer in a furnace and its comparison with experiment, Transactions of the Japan Society of Mechanical Engineers (in Japanese), Series B,Vol.64, No.626 (1998), pp.3391-3398.

Wassiljewa, A., Heat-Conduction in Gaseous Mixtures, Physik Z., Vol.5 (1904), pp. 737-742. 